Fundación

Miguel Lillo

Tucumán

Argentina

\title{
Caracterización morfológica de la semilla de Hibiscus cannabinus (Malvaceae) e influencia del tiempo de almacenamiento sobre la viabilidad
}

\author{
Morphological characterization of seed of Hibiscus cannabinus \\ (Malvaceae) and influence of the storage time on the viability
}

\author{
Bornand, Alejandra del V.* (1); Verónica S. Beltramini (1) \\ Cátedra de Botánica Morfológica. Facultad de Ciencias Agropecuarias. Félix Aldo Marrone 746, \\ Ciudad Universitaria, Universidad Nacional de Córdoba, (5000) Córdoba, Argentina. \\ * Autor corresponsal: alejandrabornand@agro.unc.edu.ar
}

\section{RESUMEN}

Hibiscus cannabinus L. (Malvaceae) "kenaf" es una especie de ciclo anual primaverootoñal, que se cultiva para la producción de fibra para papel, bioetanol de segunda generación y forraje. Los objetivos de esta investigación fueron caracterizar la morfología de la semilla de $H$. cannabinus y establecer la influencia del tiempo de almacenamiento sobre la viabilidad de dos cultivares, Endora y Tainung 1. Se realizaron observaciones de cortes histológicos y mediciones con microscopio óptico y estereoscópico. Se evaluó la germinación a los 30, 180 y 730 días después de cosecha. Las semillas de $H$. cannabinus presentan una forma asimétrica, cuneiforme y/o triangular (ca. 5,1 mm x 2,8 mm), son endospermadas y derivan de óvulos campilótropos. La cubierta seminal tiene tricomas unicelulares, eglandulares distribuidos en toda la superficie con predominio en la región del hilo. El embrión es plegado, con cotiledones conduplicados de posición axial sub tipo curvo. El episperma presenta un marcado predominio de la capa de macroesclereidas. Se encontró que las semillas de los cultivares Endora y Tainung 1, almacenadas por dos años a temperatura ambiente y con $9,5 \%$ de humedad, mantienen un alto porcentaje de germinación con valores cercanos al $80 \%$. Los resultados permiten afirmar que las semillas de "kenaf" mantienen las características morfológicas internas constantes típicas de las Malváceas, y que los cultivares estudiados presentan una alta viabilidad sostenida en el tiempo.

Palabras clave - Anatomía; cultivar; episperma; germinación; kenaf.

\footnotetext{
Ref. bibliográfica: Bornand, A. del V., Beltramini, V. S. (2021). Caracterización morfológica de la semilla de Hibiscus cannabinus (Malvaceae) e influencia del tiempo de almacenamiento sobre la viabilidad. Lilloa 58 (1): 51-62. doi: https://doi.org/10.30550/j.lil/2021.58.1/2021.04.26

> Recibido: 10 de noviembre 2020 - Aceptado: 26 de abril 2021 - Publicado en línea: 3 de mayo 2021.

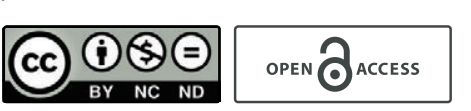

- URL de la revista: http://lilloa.lillo.org.ar

- Esta obra está bajo una Licencia Creative Commons Atribución - No Comercial - Sin Obra Derivada 4.0 Internacional.
} 


\begin{abstract}
Hibiscus cannabinus L. (Malvaceae) "kenaf" is a species with an annual spring-autumn cycle, which is cultivated for the production of fiber for paper, second generation bioethanol, and as forage. The objectives of this research are to characterize the morphology of $H$. cannabinus seed and to establish the influence of storage time on the viability of two cultivars, Endora and Tainung 1. Observations of histological sections and measurements were made with optical and stereoscopic microscopes. Germination was evaluated at 30,180 , and 730 days after harvesting. The seeds of H. cannabinus have an asymmetric, cuneiform, and/or triangular shape (ca.5.1 mm x $2.8 \mathrm{~mm}$ ), they are endospermic and derived from a campylotropous ovule. The seed coat has unicellular, eglandular trichomes distributed over the entire surface with predominance in the hilar region. The embryo is folded, with conduplicate cotyledons of axial sub-curved position. The episperm shows a marked predominance of the macrosclereid layer. The seeds of Endora and Tainung 1cultivars stored for two years at room temperature and with $9.5 \%$ humidity kept a high percentage of germination with values close to $80 \%$. These results allow to conclude that confirm that "kenaf" seeds maintain the constant, typical and internal morphological characteristics of Malvaceae, and that the studied cultivars have a high viability, sustained over time.
\end{abstract}

Keywords - Anatomy; cultivar; episperm; germination; kenaf.

\title{
INTRODUCCIÓN
}

La especie Hibiscus cannabinus L., conocida como "kenaf", pertenece a la familia Malvaceae y es originaria del sudeste y este de África (Ayadi et al., 2016). Se caracteriza por su rápido crecimiento alcanzando alturas superiores a los tres metros durante su ciclo anual primavero-otoñal, y por estar adaptada a un amplio rango de climas y tipos de suelos (Crane, 1977; Acosta Alcolea, 1999; Cosentino, D’Agosta, Copani, Testa, 2004; Falasca, Anschau, Pizarro, Cazenave, 2011). Esta especie adquirió importancia en los últimos 20 años, debido a que presenta múltiples utilidades, las fibras floemáticas de su tallo se utilizan en la obtención de pulpa celulósica para la fabricación de papel de alta calidad y también para la fabricación de productos textiles e industriales. La celulosa y hemicelulosa del tallo, se emplea para la producción de bioetanol de segunda generación. Las hojas y tallos como forraje en la alimentación de ganado bovino, ovino y porcino (Villar Gutiérrez y Amuneke, 2000; Webber, 2000; Danalatos y Archontoulis, 2002; Patane y Sortino, 2010).

Falasca et al. (2011) sostienen que el centro-norte de la provincia de Córdoba (Argentina) representa una zona apropiada para el cultivo de kenaf debido al período libre de heladas de 150 días, la temperatura media anual de $18,65^{\circ} \mathrm{C}$ y las precipitaciones medias de $800 \mathrm{~mm}$ (INTA Manfredi, 2014).

Por su parte, Pascualides, Baigorria, Rinaldi, Buffa Menghi, Bornand (2013), estudiaron el cultivar Tainung 1 y determinaron que octubre es el mes óptimo para 
la siembra de la especie. A su vez, en otros trabajos se estableció que el cultivo de kenaf presenta una alta productividad y bajo costo de producción en la zona central de Córdoba (Baigorria et al., 2009; Baigorria et al., 2010; Rinaldi et al., 2011).

Sin embargo, la introducción del cultivo de kenaf en los sistemas agrícolas de la provincia de Córdoba, no sólo depende del potencial económico y las condiciones ambientales de la zona de producción, sino también es importante conocer los aspectos morfológicos, anatómicos y cualitativos de la semilla.

Con respecto a los antecedentes sobre morfología y anatomía de las semillas de las Malváceas, Rolfs (1892) realizó una detallada descripción de la cubierta seminal de varias especies de esta familia. En este sentido, Olteanu (1933) y Reeves (1936 a, b), trabajaron con la tribu Hibisceae, específicamente con el género Abelmoschus Medik y realizaron una descripción completa de la cubierta seminal y vista interna y externa de la semilla. Por su parte, Winter (1960 a, b), analizó el desarrollo del episperma, óvulo y embrión de Abutilon theophrasti Medic. y Muneratto y Souza (2013) estudiaron la ontogenia de frutos y semillas de especies Sida rhombifolia L., Sida urens L. y Sida regnellii R. E. Fr. En relación al género Hibiscus L., en la obra de Corner (2009) se hizo una somera caracterización de la forma de la semilla y del tegumento seminal de H. cannabinus e Hibiscus esculentus L. y del mismo modo, Montejo Valdés, Muñoz, Sánchez, Gamboa (2014), realizaron un análisis topográfico de la semilla y la forma del embrión y cotiledón de Hibiscus elatus Sw. (A).

Si bien existen algunos trabajos que describen someramente la morfología de las semillas del género en estudio, no se han encontrado autores que hayan realizado una descripción detallada de la morfología de la semilla de $H$. cannabinus.

Con respecto a la calidad fisiológica, entre las contribuciones para kenaf, pueden mencionarse los trabajos de Cook y Smart (1994), Meints y Smith (2003), Mollah, et al. (2015) quienes observaron que el porcentaje de germinación para esta especie fue superior al $80 \%$. Además, Toole, Toole, Nelson (1959) y Meints y Smith (2003) estudiaron la viabilidad de distintos cultivares de kenaf en diferentes tiempos y condiciones de almacenamiento, y determinaron que el porcentaje de germinación de las semillas a los dos años de cosecha fue cercano al $80 \%$, valor que podía disminuir si las condiciones de humedad de la semilla no eran las adecuadas.

Teniendo en cuenta lo anteriormente expuesto, los objetivos de este trabajo fueron caracterizar la morfología de la semilla de $H$. cannabinus y establecer la influencia del tiempo de almacenamiento sobre la viabilidad de los cultivares Endora y Tainung 1 .

\section{MATERIALES Y MÉTODOS}

\section{Material vegetal}

Se trabajó con semillas de dos cultivares de $H$. cannabinus, Endora y Tainung 1, provenientes de ensayos ubicados en un predio al Noroeste de la ciudad de Córdoba, Argentina en el año 2015 (Lat. Sur $31^{\circ} 24^{\prime} 08.1^{\prime \prime}$ y Long. Oeste 64 $15^{\prime}$ ).

Se realizó una trilla manual y se determinó el contenido de humedad de las semillas según Reglas ISTA (2015). El cultivar Tainung 1 presentó 9,53\% y Endora $9,44 \%$ de humedad respectivamente. 
Las semillas, una vez limpias (sin impurezas), fueron acondicionadas en bolsas de papel madera a temperatura ambiente $\left(20 \pm 3^{\circ} \mathrm{C}\right)$.

\section{Caracterización morfológica de la semilla}

El análisis topográfico de embrión, endosperma y cubierta seminal, se realizó sin hacer distinción de cultivares, ya que los mismos sólo se diferencian por la forma de sus hojas y la sensibilidad al fotoperiodo (Oliveros, Ponz, Manzanares, Tenorio, Ayerbe, 2000). En este sentido, no se esperan diferencias anatómicas entre los mismos, porque están emparentados y los caracteres son constantes dentro de una misma especie. Sin embargo, entre cultivares el tamaño puede variar cuantitativamente, por ello, se midieron 100 semillas de cada uno con un calibre digital y los resultados se expresaron en milímetros.

Las características externas de la semilla se analizaron según los criterios y la terminología propuesta por Esau (1982), mientras que, para las características internas generales de la semilla, en particular las del embrión y endosperma, se siguió la descripción de Martin (1946). Para el análisis topográfico de la semilla y de las características del embrión y endosperma, se realizaron cortes en los planos transversal y sagital que fueron observados con estereomicroscopio Zeiss Stemi DV4. La determinación del color de la cubierta seminal se realizó teniendo en cuenta la carta de colores de Munsell (2000). En cuanto a la descripción anatómica de la cubierta seminal y del endosperma se realizaron cortes transversales a mano alzada. Las secciones se tiñeron con safranina ( $1 \mathrm{~g}$ de safranina diluida en $100 \mathrm{ml}$ de etanol al 50\%) (D’ambrogio de Argüeso, 1986). Los distintos estratos se midieron con micrómetro de ocular adosado a un microscopio óptico binocular Modelo Primo Star Zeiss. Se registraron imágenes mediante cámara digital Nikon Coolpix S 10.

\section{Ensayo de germinación y viabilidad en el tiempo}

Se realizaron ensayos de germinación en 3 tiempos distintos de almacenamiento, a los 30, 180 y 730 días después de cosecha (DDC). Se colocaron a germinar cuatro repeticiones de 100 semillas de cada cultivar, Endora y Tainung 1, en rollos de papel humedecido con agua destilada, y se ubicaron en bandejas plásticas envueltas en bolsas de polietileno para mantener la humedad. Se acondicionaron en cámaras de germinación de manera totalmente aleatorizada, reguladas a temperatura alternante de $20-30^{\circ} \mathrm{C}$, con 8 horas de oscuridad y 16 horas de luz. Al cabo de 8 días se determinó el porcentaje de germinación (ISTA, 2015).

\section{Análisis y diseño estadístico}

Los resultados de las variables cuantitativas se sometieron a un Análisis de la Varianza (ANOVA) y se determinaron las diferencias mínimas significativas entre las medias (DMS) de los tratamientos con el test DGC a un nivel de significancia de 0,05 a través del programa Infostat (Di Rienzo et al., 2018). 


\section{RESULTADOS Y DISCUSIÓN}

\section{Caracterización de la semilla}

Las semillas se caracterizan por ser asimétricas, reniformes, cuneiformes y/o triangulares (Fig. 1).

La tabla 1 muestra los valores promedios y errores estándares de las medidas de las semillas de los dos cultivares de kenaf. No se encontraron diferencias significativas $(p>0,05)$ entre cultivares en el largo $5,1 \mathrm{~mm}( \pm 0,04)$ y ancho $2,8 \mathrm{~mm}( \pm$ $0,02)$. Dada su asimetría, en una vista lateral se tomaron dos alturas. La altura 1 , tomada desde el extremo del lóbulo radicular hasta la base, fue para el cultivar Endora significativamente mayor ( $\mathrm{p}>0,05)$, con un valor de $5,22 \mathrm{~mm}( \pm 0,03)$, mientras que Tainung 1 midió $4,87 \mathrm{~mm}( \pm 0,03)$. Por otro lado, la altura 2 , tomada desde la mitad de la longitud hasta la base, el cultivar Endora midió $3,24 \mathrm{~mm}( \pm 0,02)$, valor significativamente mayor que Tainung 1 con $3,12 \mathrm{~mm}( \pm 0.02)$ (Fig. 2 A y B). La medida del largo de la semilla es similar a la observada por Corner (2009) en $H$. cannabinus $e$ H. esculentus. Con respecto al color, se determinó un gris amarronado oscuro (5YR, valor 4, intensidad 1). En la superficie de la cubierta seminal se
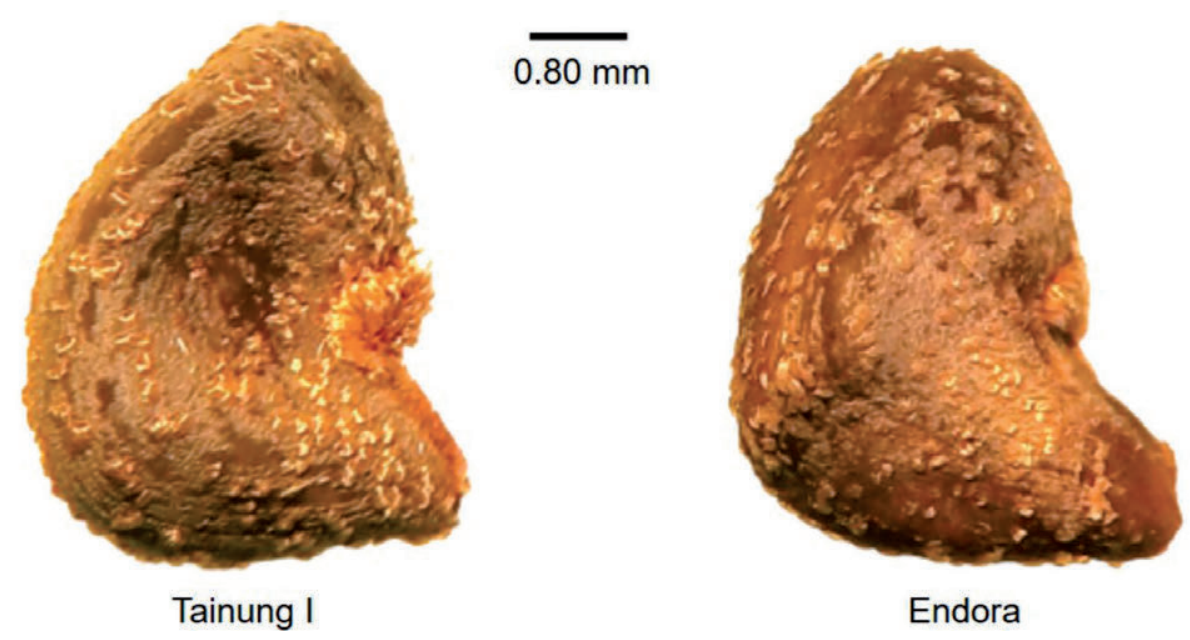

Fig. 1. Vista externa de la semilla de los dos cultivares de H. cannabinus, Tainung I y Endora.

Fig. 1. External view of the seed of the two cultivars of $H$. cannabinus, Tainung I and Endora.

Tabla 1. Valores promedios y error estándar del largo, el espesor y la altura de las semillas de dos cultivares de $H$. cannabinus en milímetros.

Table 1. Average values and standard error of the length, thickness and height of the seeds of two cultivars of $H$. cannabinus in millimeters.

\begin{tabular}{l|c|c|c|c} 
Cultivar & Largo (I) & Espesor (e) & \multicolumn{2}{|c}{ Alturas } \\
\cline { 1 - 4 } & & & $\begin{array}{c}\text { Extremo radicular } \\
\text { (a) }\end{array}$ & $\begin{array}{c}\text { Región hilar } \\
(\mathrm{b})\end{array}$ \\
\hline Endora & $5,12 \pm 0,04 \mathrm{a}$ & $2,81 \pm 0,02 \mathrm{a}$ & $5,22 \pm 0,03 \mathrm{~b}$ & $3,24 \pm 0,02 \mathrm{~b}$ \\
\hline Tainung 1 & $5,11 \pm 0,04 \mathrm{a}$ & $2,79 \pm 0,02 \mathrm{a}$ & $4,87 \pm 0,03 \mathrm{a}$ & $3,12 \pm 0,02 \mathrm{a}$ \\
\hline
\end{tabular}



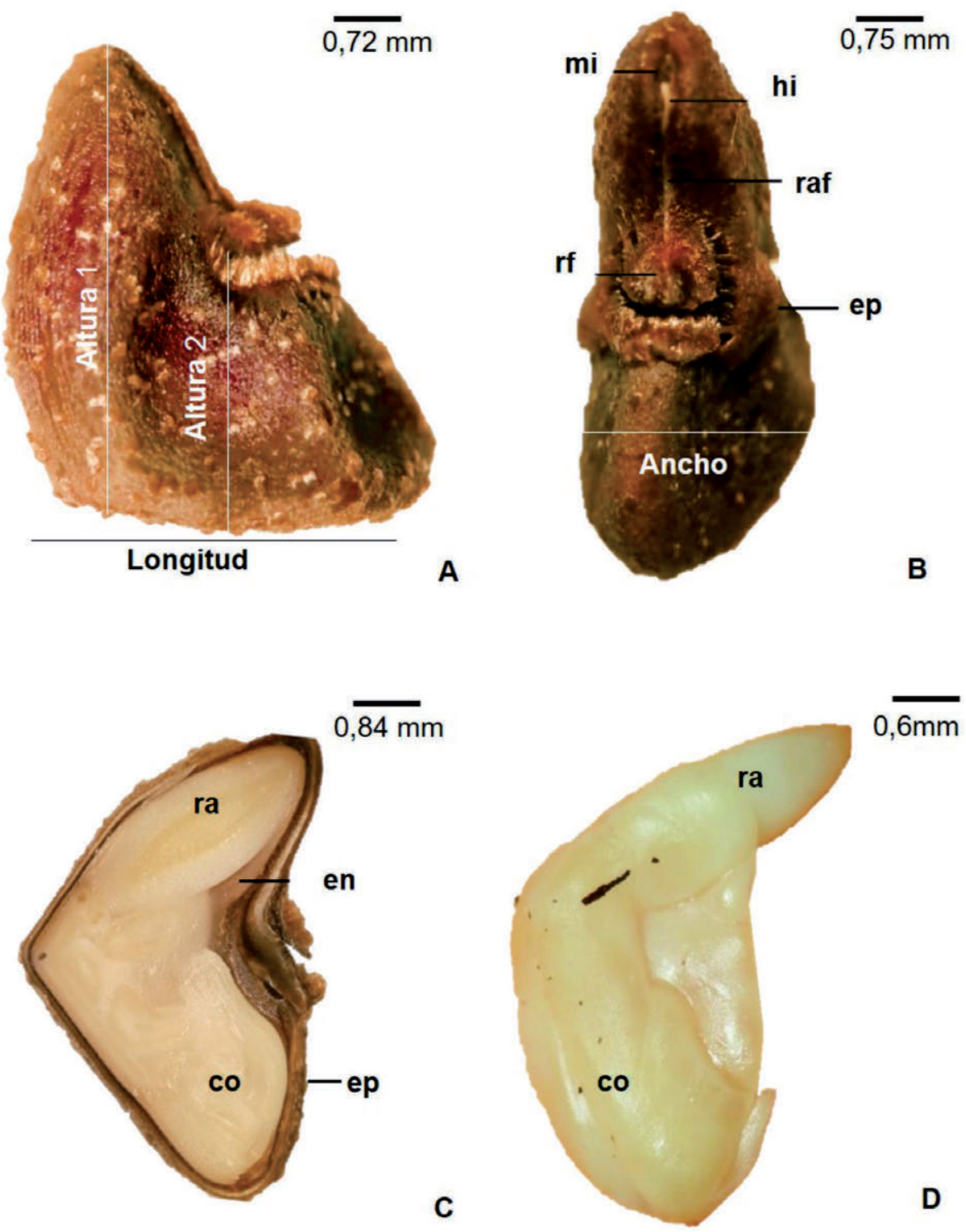

Fig. 2. Características morfológicas de la semilla de $H$. cannabinus. A) Vista externa lateral. B) Vista externa frontal. C) Corte sagital de la semilla. D) Embrión.

Abreviaturas: co, cotiledones; en, endosperma; ep, episperma; hi, hilo; mi, micrópilo; ra, radícula; raf, rafe; rf, restos de funículo.

Fig. 2. Morphological characteristics of the $H$. cannabinus seed. A) Lateral external view. B) Front external view. C) Sagittal section of the seed. D) Embryo.

Abbreviations: co, cotyledons; en, endosperm; ep, episperm; hi, hilum; mi, micropyle; ra, radicle; raf, raphe; rf, remains of funiculus.

observaron tricomas dispuestos en placas, con mayor densidad en la región hilar y calazal (Fig. 3 A y D).

De acuerdo a su forma, la posición del embrión y las cicatrices presentes en el episperma, la semilla deriva de un óvulo campilótropo, característica mencionada también por Olteanu (1933), Reeves (1936 a,b), Winter (1960a), Poljakoff-Mayber, Somers, Werker, Gallagher (1992), Muneratto y Souza (2013) en algunas especies de Malváceas y Corner (2009) y Montejo Valdés et al. (2014) en el género Hibiscus. 

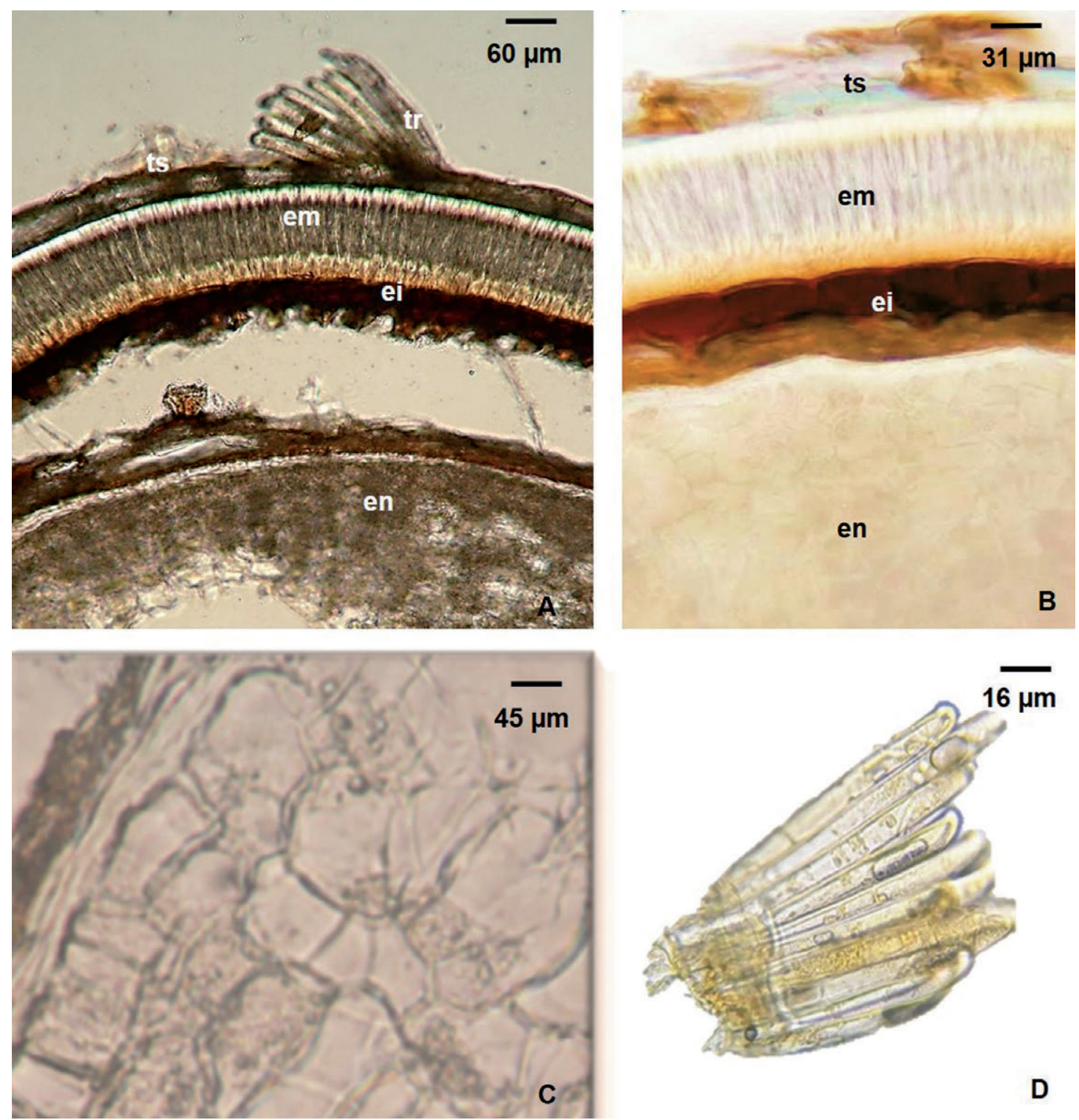

Fig. 3. Anatomía de la cubierta seminal de $H$. cannabinus. A) Corte transversal de la cubierta seminal con aumento. B) Corte transversal de la cubierta seminal. C) Detalle de las células del endosperma. D) Tricomas. Abreviaturas: em, empalizada-macroesclereidas; ei, epidermis interna; en, endosperma; tr, tricoma; ts, testa.

Fig. 3. Anatomy of the seed coat of $H$. cannabinus. A) Cross section of the seed coat. B) Cross section of the seminal coat. C) Detail of the endosperm cells. D) Trichomes. Abbreviations: em, empalisademacrosclereid; ei, internal epidermis; en, endosperm; tr, trichome; ts, testa.

En una vista frontal de la semilla (Fig. 2 B), se aprecia desde el extremo superior hacia abajo: el lóbulo radicular aguzado donde se hace visible el micrópilo de forma circular, el hilo longitudinal y el rafe, ambos de color blanquecino, y por último se observan restos del funículo con numerosos tricomas que cubren la región calazal y que a menudo se desprenden. En términos generales estas características concuerdan con lo mencionado por Olteanu (1933), Poljakoff-Mayber et al. (1992), Montejo Valdés, Sánchez Rendón, Muñoz García (2011) y Muneratto y Souza (2013) en distintos estudios que abarcan la morfología de semillas de Malváceas. 
Tal como lo nombra Reeves, 1936b, para el género Hibiscus y Montejo Valdés et al. (2014) en $H$. elatus, el embrión es de posición axial sub tipo curvo y continuo, sus órganos están diferenciados y de acuerdo a la clasificación establecida por Baskin y Baskin (1998 y 2007) basado en Martin (1946), es un embrión plegado característico de la familia. Se observó que los cotiledones son del tipo conduplicados, se doblan longitudinalmente, se envuelven entre sí y ambos a la radícula.

Como fue determinado para otras semillas de especies de Malváceas (Olteanu, 1933; Reeves, 1936a, b; Winter, 1960b; Chandra y Bhatnagar 1975; Poljakoff-Mayber et al., 1992; Muneratto y Souza, 2013; Arambarri, 2018), la semilla de H. cannabinus es endospermada. El endosperma en esta especie se encuentra como una capa delgada que envuelve al embrión, siendo de mayor espesor en la hendidura de la región hilar y calazal, donde el embrión se curva (Fig. 2 C y D). Además, se registró la presencia de endosperma entre los pliegues de los cotiledones, lo que coincide con lo mencionado por Poljakoff-Mayber et al. (1992) en Kosteletzkya virginica (L.) Presl. (Malvaceae).

\section{Anatomía de la cubierta seminal y el endosperma}

El episperma de $H$. cannabinus (Fig. 3 A-B), está compuesto por 2 capas, una externa o testa (ca. $26 \mu \mathrm{m}$ de espesor) y la otra interna o tegmen (ca. $155 \mu \mathrm{m}$ de espesor). La capa externa contiene células epidérmicas de paredes delgadas que se disponen de forma aplastada. Asimismo, se observan en la superficie abundantes tricomas eglandulares, unicelulares, aguzados con medidas que oscilan entre los 90 y $132 \mu \mathrm{m}$ de largo, se disponen en placas y se encuentran en mayor densidad en la región del hilo. La mayoría de las células del episperma y tricomas se presentan pigmentados de color marrón (Fig. 3 D) (Corner, 2009). Por otro lado, la epidermis externa del tegmen (ca. 98,78 $\mu \mathrm{m}$ de espesor) presenta una capa de macroesclereidas (células de Malpighi o en empalizada) dispuestas de forma compacta, con células alargadas y de paredes gruesas lignificadas, evidenciándose en la parte superior una línea lúcida continua. La epidermis interna (56,25 $\mu \mathrm{m}$ de espesor), se compone de un tejido de dos a tres capas de células con paredes gruesas y redondeadas, con contenido celular color marrón oscuro. A su vez, adnato al endosperma, se evidencia una capa cuticular de tonalidad más clara.

Lo expuesto anteriormente coincide con lo investigado por Corner (2009) en $H$. cannabinus, quien además estableció que la cubierta de las semillas de las Malváceas tiene una estructura uniforme y destacó a la empalizada del tegmen como una característica principal. Además, existen otros trabajos de la cubierta seminal de Malváceas (Rolfs, 1892; Olteanu 1933; Reeves, 1936 a, b; Winter, 1960b; Vaughan, 1970; Poljakoff-Mayber et al., 1992; Muneratto y Souza, 2013), en los que se han determinado patrones similares a los encontrados en kenaf.

El endosperma presentó una gran variabilidad en la forma de sus células, las cuales pueden ser circulares, rectangulares y cuadradas con paredes celulares delgadas (Fig. 3 C). Esto coincide con lo observado por Winter (1960b), Poljakoff-Mayber et al. (1992) y Muneratto y Souza (2013), quienes además encontraron gránulos de almidón en el endosperma de algunas especies de malváceas. 


\section{Ensayo de germinación estándar en tres tiempos de almacenamiento}

La tabla 2 muestra los resultados de los ensayos de germinación realizados en tres tiempos diferentes, a los 30, 180 y 730 días después de la cosecha (DDC).

Tabla 2. Porcentaje de germinación de dos cultivares de $H$. cannabinus en diferentes tiempos de almacenamiento.

Table 2. Germination percentage of two $H$. cannabinus cultivars at different storage times.

\begin{tabular}{l|c|c|c}
\hline Cultivar & 30 DDC & 180 DDC & 730 DDC \\
\hline Endora & $87,25 \pm 2,3 \mathrm{~b}$ & $88,75 \pm 2,3 \mathrm{~b}$ & $87,00 \pm 2,3 \mathrm{~b}$ \\
\hline Tainung 1 & $73,75 \pm 2,3 \mathrm{a}$ & $84,50 \pm 2,3 \mathrm{~b}$ & $79,75 \pm 2,3 \mathrm{a}$ \\
\hline
\end{tabular}

El cultivar Endora presentó una germinación significativamente mayor que el cultivar Tainung 1 a los 30 y $730 \mathrm{DDC}(\mathrm{F}=5,78 ; \mathrm{GL}=5, \mathrm{P}<0,05)$. En este último cultivar se observó un mayor porcentaje de germinación a los 180 DDC, comparado con los 30 y 730 DDC.

Este comportamiento se asemeja al observado por Toole et al. (1959) y Meint y Smith (2003), quienes determinaron que las semillas con 6 a 12 meses de almacenamiento a temperatura ambiente y con 8 a 12\% de HR, aumentaron el porcentaje de germinación. A su vez, estos valores concuerdan con los estudiados por otros autores en distintos cultivares de kenaf, entre ellos Cook y Smart (1994), quienes trabajaron con el cultivar Tainung 1 y a los seis meses de almacenamiento el porcentaje de germinación fue de $89,1 \%$, valor parecido al registrado en el presente trabajo. Del mismo modo, Olasoji et al. (2012) obtuvieron 85\% de germinación en Tainung 1 y Mollah et al. (2015) arribaron a porcentajes de germinación superiores al $80 \%$ en otros cultivares de kenaf.

Por otro lado, se observó que las semillas con dos años de almacenamiento a temperatura ambiente y $9,5 \%$ de humedad, mantienen su viabilidad con valores cercanos al 80\%. Estos resultados concuerdan con Toole et al. (1959), quienes determinaron que las semillas se pueden almacenar a temperatura ambiente $\left(21^{\circ} \mathrm{C}\right)$ y con $8 \%$ de humedad por 66 meses y 12\% de humedad por 24 meses. Del mismo modo, Meints y Smith (2003), establecieron que la semilla de kenaf almacenada hasta 4 años a $10^{\circ} \mathrm{C}$ retiene tasas de germinación aceptables para uso comercial cercanas al $80 \%$.

\section{CONCLUSIÓN}

En sentido general las semillas mantienen las características morfológicas internas constantes típicas de las Malváceas. En la anatomía de la cubierta seminal se destaca un marcado predominio de la capa de macroesclereidas. En relación a la influencia del tiempo de almacenamiento sobre la viabilidad, se demuestra que los 2 cultivares, Endora y Tainung 1, tienen una alta viabilidad sostenida en el tiempo, con valores cercanos al $80 \%$. Además, se destaca que el cultivar Endora mantiene los valores 
de germinación sin variaciones en los dos años estudiados, este comportamiento se podría explicar por el mayor tamaño registrado en las alturas de la semilla. Se considera que la información lograda de la morfoanatomía de $H$. cannabinus descripta por primera vez en este trabajo, es un aporte para continuar con el estudio de la semilla de esta especie.

\section{AGRADECIMIENTOS}

Las autoras agradecen a la Ing. Agr. Ana Lía Pascualides por su acompañamiento y los aportes realizados en el manuscrito y al Laboratorio de Análisis de Semillas (LASIDYS) de la Facultad de Ciencias Agropecuarias de la Universidad Nacional de Córdoba, por brindar los espacios para el trabajo de laboratorio. Agradecemos a los revisores anónimos por las sugerencias para mejorar el presente trabajo.

\section{BIBLIOGRAFÍA}

Acosta Alcolea, G. (1999). Aspectos generales del kenaf (Hibiscus cannabinus), en Cuba. Revista de la Universidad de la mixteca. Temas de Ciencia y Tecnología $N^{o}$ 7: 3-31.

Arambarri, A. M. (2018). Diásporas y Semillas. Facultad de Ciencias Agrarias y Forestales de la Universidad Nacional de la Plata.

Ayadi, R., Hanana, M., Mzid, R., Hamrouni, L., Khouja, M. 1. y Salhi Hanachi, A. (2016). Hibiscus cannabinus L. "Kenaf »: A Review Paper. Fournal of Natural Fibers 14 (4): 1-19. DOI: 10.1080/15440478.2016.1240639

Baigorria, M. del C., Buffa Menghi, M. N., Rinaldi, G., Serena, J., Videla, J. y Bornancini, J. (2009). Estudio económico de la factibilidad de incorporar kenaf en la rotación de cultivos anuales en Villa del Totoral, Provincia de Córdoba, Argentina. XL Reunión Anual de la Asociación Argentina de Economía Agraria. Bahía Blanca.

Baigorria, M. del C., Pascualides, A. L., Buffa Menghi, M. N., Rinaldi, G. y Serena, J. (2010). Kenaf: cultivo alternativo para la fabricación de papel en la región semiárida de Córdoba. Facultad de Ciencias Agropecuarias. Universidad Nacional de Córdoba. Marca Líquida ISSN-16689224.

Baskin, J. M. y Baskin, C. C. (1998). Seeds: Ecology, biogeography and evolution of dormancy and germination. San Diego, CA., USA. Academy Press.

Baskin, J. M. y Baskin, C. C. (2007). A revision of Martin's seed classification system, with particular reference to his dwarf-seed type. Seed Science Research 17: 11-20.

Chandra, S. y Bhatnagar, S. P. (1975). Seed development of Abelmoschus esculentus (Malvaceae). Plant systematic and evolution 123: 255-262.

Cook, C. G. y Smart, J. R. (1994). Seed quality evaluation of eigth kenaf cultivars. Industrial Crops and Products 3: 213-216.

Corner, E. J. H. (2009). The Seeds of Dicotyledons. Cambridge. University Press. New York. 
Cosentino, S. L., D’Agosta, G. M., Copani, V. y Testa, G. (2004). Yield and development of kenaf (Hibiscus cannabinus L.) crop in relation to genotype, sowing time and plant population in Mediterranean environment. In: Van Swaalj W.P.M., Fjalistrom T., Helm P., Grassi A. (Ed.), Biomass for Energy, Industry and Climate Protection.

Crane, J. (1977). Kenaf fiber plant, rival of yute. Economic Botany 1 (3): 334-350.

Esau, K. (1982). Anatomía de las plantas con semillas. Ed. Hemisferio Sur. Buenos Aires.

Danalatos, N. G. y Archontoulis, S. V. (2002). Sowing time and plant density effects on growth and biomass productivity of two kenaf varieties in central Greece. In: J.Janick and A. Whipkey (eds.). ASHS Press, Alexandria, VA. Kenaf Yield.

D’ambrogio de Argüeso, A. (1986). Manual de técnicas en histología vegetal. Editorial Hemisferio Sur S.A.

Di Rienzo, J. A., Casanoves, F., Balzarini, M. G., González, L., Tablada, M. y Robledo, C. W. (2018). Grupo InfoStat, FCA, Universidad Nacional de Córdoba, Argentina. http://www.infostat.com.ar

Falasca, S. L., Anschau, A., Pizarro, M. J. y Cazenave, G. (2011). Las posibilidades de cultivo de kenaf (Hibiscus cannabinus L.) como productor de biomasa en Argentina. Cuarto Congreso Nacional y Tercer Congreso Iberoamericano Hidrógeno y Fuentes Sustentables de Energía.

INTA Manfredi. (2014). Información meteorológica mensual de la EEA Manfredi. https://inta.gob.ar/documentos/informacion-meteorologica-mensual-de-la-eeamanfredi

International Seedling Test Association (ISTA). (2015). International Rules for Seed Testing. The International Seed Testing Association (ISTA). Switzerland.

Martin, A. C. (1946). The comparative internal morphology of seeds. American Midland Naturalist 3: 513-660.

Meints, D. D. y Smith, C. A. (2003). Kenaf seed storage duration on germination, emergence, and yield. Industrial Crops and Products 17: 9-14.

Mollah, A. F., Rahman, M. M., Tareq, M. Z., Hasan, M. M. y Hoque, A. B. M. Z. (2015). Yield and quality of kenaf seed as influenced by de-topping and spacing under transplanting method. International Fournal of Applied Sciences and Biotechnology 3 (4): 626-634.

Montejo Valdés, L. A., Sánchez Rendón, J. A. y Muñoz García, B. C. (2011). Caracterización morfológica de frutos, semillas y plántulas de Talipariti elatum (Malvaceae). Acta Botánica Cubana. N ${ }^{\circ}$ 210: 45-49.

Montejo Valdés, L. A., Muñoz, B. C., Sánchez, J. A. y Gamboa, A. (2014). Variabilidad seminal entre las especies de un bosque siempre verde tropical de la Sierra del Rosario, Cuba. Bosque 35 (1): 37-47.

Muneratto, J. C. y Souza, L. A. (2013). Ontogenia del fruto (pericarpio y semilla) de especies de Sida. Gayana Botánica 70 (1): 44-56.

Munsell soil color charts. (2000). Revised washable edition. Ed. Gretagmacbeth. New Windsor, US.

Olasoji, O. J., Aluko, A. O., Adeniyan, O. N, Olanipekun, S. O., Olosunde, A. A. y Okoh, J. O. (2012). Effect of time of harvested on physiological maturity and 
kenaf (Hibiscus cannabinus) seed quality. African Fournal of Plant Science 6 (10): 282-289.

Oliveros, R., Ponz, R., Manzanares, M., Tenorio, J. L. y Ayerbe, L. (2000). El Kenaf. Un cultivo agrícola para la producción de fibras anuales. Agricultura revista agropecuaria: 748-753. ISSN: 0002-1334.

Olteanu, R. (1933). Etude monographique sur Hibiscus esculentus (Abelmoschus esculentus L. Mey.). Societe polygraphique.

Pascualides, A. L.; Baigorria, M. C, Rinaldi, G. V., Buffa Menghi, M. N. y Bornand, A. del V. (2013). Evaluación de dos fechas de siembra de Hibiscus cannabinus L. "kenaf" (Malvaceae) en Villa del Totoral, Córdoba, Argentina. Agronomía E Ambiente. Tomo 33 (1-2): 31-42.

Patane, C. y Sortino, O. (2010). Seed yield in kenaf (Hibiscus cannabinus L.) as affected by sowing time in South Italy. Industrial Crops and Products 32: 381-388.

Poljakoff-Mayber, A., Somers, G. F., Werker, E. y Gallagher, J. L. (1992). Seeds of Kosteletzkya virginica (Malvaceae): their structure, germination and salt tolerance. American journal of botany 79: 249-256.

Reeves, R. G. (1936a). Comparative anatomy of the seeds of cottons and other malvaceous plants. I. Malveae and Ureneae. American Fournal of Botany 23: 291-296.

Reeves, R. G. (1936b). Comparative anatomy of the seeds of cottons and other malvaceous plants. II. Hibisceae. American Fournal of Botany 23: 394-405.

Rinaldi, G., Buffa Menghi, M. N., Pascualides, A. L., Baigorria, M. C., Serena, J., Bornand, A. V. y Malpiedi, A. (2011). Análisis de los componentes del rendimiento de fibra y su incidencia económica para dos densidades de siembra en Hibiscus cannabinus L. "kenaf" y Crotalaria juncea L. "crotalaria de fibra" en la provincia de Córdoba. VII Jornadas Interdisciplinarias de Estudios Agrarios (CIEA) de la Facultad de Ciencias Económicas, UBA. ISSN 1851-3794.

Rolfs, P. H. (1892). The seed coats of Malvaceae. Botanical Gazette 17: 33-39.

Toole, E. H., Toole, V. K. y Nelson, E. G. (1959) Preservation of hemp and kenaf seed. USDA Technical Bulletins.

Vaughan, J. G. (1970). The structure and utilization of oil seeds. Chapman \& Hall Ltd., London. En Corner, E. J. H. 1976. The seeds of the dicotyledons. Vol 1. Cambridge University Press.

Villar Gutiérrez, J. C. y Amuneke, E. J. (2000). Utilización de kenaf (Hibiscus cannabinus L.) en la producción de diferentes tipos de pastas. Congreso Iberoamericano investigación en celulosa y papel. www.riadicyp.org.ar

Webber, C. L. (2000). Kenaf (Hibiscus cannabinus L.): New horizons for an ancient crop. Proceedings International Kenaf Association Conference Oklahoma City, OK.

Winter, D. (1960a). The development of the seed of Abutilon theophrasti. I Ovule and embryo. American journal of botany 47 (1): 8-14.

Winter, D. (1960b). The development of the seed of Abutilon theophrasti. II Seed coat. American journal of botany 47 (3): 157-162. 